\title{
Multiscale Disordered Porous Fibers for Self-Sensing and Self-Cooling Integrated Smart Sportswear
}

DOI:

10.1021/acsnano.9b06899

\section{Document Version}

Accepted author manuscript

Link to publication record in Manchester Research Explorer

\section{Citation for published version (APA):}

Hu, X., Tian, M., Xu, T., Sun, X., Sun, B., Sun, C., Liu, X., Zhang, X., \& Qu, L. (2020). Multiscale Disordered Porous Fibers for Self-Sensing and Self-Cooling Integrated Smart Sportswear. ACS Nano, 14(1), 559-567. https://doi.org/10.1021/acsnano.9b06899

\section{Published in:}

ACS Nano

\section{Citing this paper}

Please note that where the full-text provided on Manchester Research Explorer is the Author Accepted Manuscript or Proof version this may differ from the final Published version. If citing, it is advised that you check and use the publisher's definitive version.

\section{General rights}

Copyright and moral rights for the publications made accessible in the Research Explorer are retained by the authors and/or other copyright owners and it is a condition of accessing publications that users recognise and abide by the legal requirements associated with these rights.

\section{Takedown policy}

If you believe that this document breaches copyright please refer to the University of Manchester's Takedown Procedures [http://man.ac.uk/04Y6Bo] or contact uml.scholarlycommunications@manchester.ac.uk providing relevant details, so we can investigate your claim.

\section{OPEN ACCESS}


This document is confidential and is proprietary to the American Chemical Society and its authors. Do not copy or disclose without written permission. If you have received this item in error, notify the sender and delete all copies.

\section{Multiscale Disordered Porous Fibers for Self-Sensing and Self-Cooling Integrated Smart Sportswear}

\begin{tabular}{|c|c|}
\hline Journal: & ACS Nano \\
\hline Manuscript ID & nn-2019-06899a.R2 \\
\hline Manuscript Type: & Article \\
\hline $\begin{array}{r}\text { Date Submitted by the } \\
\text { Author: }\end{array}$ & $\mathrm{n} / \mathrm{a}$ \\
\hline Complete List of Authors: & $\begin{array}{l}\text { Hu, Xili; Qingdao University } \\
\text { Tian, Mingwei; Qingdao University, } \\
\text { Xu, Tailin; University of Science and Technology Beijing } \\
\text { Sun, Xuantong; The University of Manchester } \\
\text { Sun, Bing; Sinopec Research Institute of safety Engineering } \\
\text { Sun, Chengcheng; Qingdao University } \\
\text { Liu, Xuqing; The University of Manchester } \\
\text { Zhang, Xueji; Shenzhen University Health Science Center } \\
\text { Qu, Lijun; Qingdao University, }\end{array}$ \\
\hline
\end{tabular}

\section{SCHOLARONE ${ }^{m}$ Manuscripts}




\title{
Multiscale Disordered Porous Fibers for Self-Sensing and Self-Cooling Integrated Smart Sportswear
}

\author{
Xili Hu$u^{\mathrm{a}, 1}$, Mingwei Tian ${ }^{\mathrm{a}, 1, *}$, Tailin $\mathrm{Xu}^{\mathrm{b},{ }^{*}}$, Xuantong Sun ${ }^{\mathrm{d}}$, Bing Sun ${ }^{\mathrm{c}}$, Chengcheng \\ Sun $^{\mathrm{a}}$, Xuqing Liud, Xueji Zhang, ${ }^{\mathrm{e},}$, Lijun $\mathrm{Qu}^{\mathrm{a},{ }^{*}}$ \\ a Research Center for Intelligent and Wearable Technology, College of Textiles and Clothing, State \\ Key Laboratory of Bio-Fibers and Eco-Textiles, Collaborative Innovation Center for Eco-Textiles of \\ Shandong Province, Qingdao University, Qingdao, Shandong, 266071, P.R. China \\ ${ }^{\mathrm{b}}$ Research Center for Bioengineering and Sensing Technology, University of Science and \\ Technology Beijing, 30 Xueyuan Road, Beijing 100083, P. R. China \\ c Sinopec Research Institute of safety Engineering, Qingdao 266071, China \\ d School of Materials, The University of Manchester, Oxford Road, Manchester, \\ M13 9PL, U.K. \\ e School of Biomedical Engineering, Shenzhen University Health Science Center, Shenzhen, \\ Guangdong 518060, P. R. China
}

\footnotetext{
${ }^{1}$ These authors equally contributed to this work.

Corresponding author: mwtian@qdu.edu.cn (Prof. Tian), xutailin@ustb.edu.cn (Prof. Xu), zhangxueji@ustb.edu.cn (Prof. Zhang), lijunqu@qdu.edu.cn (Prof. Qu)
} 


\begin{abstract}
Smart clothing has demonstrated potential applications in a wide range of wearable fields for human body monitoring and self-adaption. However, current wearable sensors often suffer from seamlessly integrating with normal clothing, restricting sensing ability and the experience for wearing. Here, an integrated smart clothing is fabricated by employing multiscale disordered porous elastic fibers as sensing units, which shows the capability of inherently autonomous self-sensing (i.e., strain and temperature sensing) and self-cooling. The multiscale disordered porous structure of the fibers contributes to the highly transparency of mid-infrared human body radiation and backscatter visible light, which allows the microenvironment temperature between skin and clothing dropping of at least $\sim 2.5^{\circ} \mathrm{C}$ than cotton fabrics. After the capillaryassisted adsorption of graphene inks, the modified porous fibers could also possess realtime strain and temperature sensing capacities with high gauge factor (GF) and thermal coefficient of resistance (TCR). As a proof of concept, the integrated smart sportswear achieved measuring body temperature, and tracking large-scale limp movements and collecting subtle human physiological signals, along with the intrinsic self-cooling ability.
\end{abstract}

KEYWORDS: multiscale disordered porous structure, smart clothing, thermal management, infrared radiation transparency, tensile strain sensor, temperature sensor. 
Smart clothing is a group of advanced conceptual product which can equip self(sensing, actuating, adaption, healing, etc.) smart elements onto clothing to monitor aspects of human health and wellness, location, positioning, predominant posture, walking speed, and so forth. ${ }^{1-4}$ As a crucial fundamental unit, emerging wearable sensing materials, such as strain sensors,${ }^{5}$ temperature sensors, ${ }^{6}$ humidity and moisture sensors, ${ }^{7}$ have considerably attracted numerous attentions on how to fabricate flexible strain sensors with high sensitivity, wide sensing range and durability. To date, the integrating routes of sensors on clothing is typically determined by structures and materials of the sensors, directly adhered onto human skin $^{8}$ or encapsulated into fabrics, ${ }^{9}$ which results in unsatisfied wear experience. Aside from efficient real time sensing properties of the sensors, the compatibility with clothing substrate and wearing experience (such as comfortable, durable, resistant to regular textile maintenance processes) are also essential for smart clothing. ${ }^{10}$

Most recently, flexible textile-based sensors have been prospective candidates for smart clothing due to their comfortability. ${ }^{11}$ For instance, several common textiles with multidimensional structures have been attempted to act as the textile-based sensors, such as, 1D fibers, ${ }^{12}$ filaments ${ }^{13}$ and yarns, ${ }^{14} 2 \mathrm{D}$ wove ${ }^{15}$ and knitted ${ }^{16}$ fabrics and films, ${ }^{17}$ 3D fabrics ${ }^{18}$ etc. In fact, despite the intrinsic flexible property is obvious improved, these sensors commonly need to adhere onto clothes or skin with the aid of silicone-based elastomers (e.g. PDMS) or rubbers which might lead to stuffiness wear experience. ${ }^{8}$ Which means, the mainly constraints that restrict the wearable application still exist. It should be noted that an ideal textile-based sensing material should be a part 
of clothes, rather than accessories, and do not need any hard, rigid components. Thus, it is highly desirable to simplify the integration of wearable sensors and our clothes, or even make them all in one clothing intrinsically.

Herein, we fabricated multiscale disordered porous elastic polyurethane (MPPU) fibers with self-sensing and self-cooling properties via the microfluidic spinning. We accomplish real-time sensation and functions of clothing with the aid of graphene nanosheets onto MPPU fibers, as well as outstanding self-cooling property endowed by multiscale disordered porous structure of the fibers. The multiscale disordered pores (nano-pores: $\sim 469 \mathrm{~nm}$, micro-pores: $\sim 34 \mu \mathrm{m}$ ) in PU fibers contribute to the highly transparency of mid-infrared human body radiation and backscatter visible light, which allows for the microenvironment temperature between skin and clothing drops $\sim 2.5^{\circ} \mathrm{C}$ than that of cotton fabrics. Moreover, the accompanying graphene doped PU (G@MPPU) fibers can achieve real-time self-sensing (i.e., strain and temperature sensing) due to the high gauge factor (GF) and thermal coefficient of resistance (TCR). Therefore, such multifunctional PU fibers can be fabricated into smart sportswear.

\section{RESULTS AND DISCUSSION}

Figure 1a depicts the formation mechanism of multiscale disordered porous polyurethane (MPPU) fibers through the phase-separate-based microfluidic spinning method. The poly(methyl methacrylate) (PMMA) chip was designed with a pattern of three inlet channels and a coaxial flow channel (Figure 1b, Figure S1) for the fiber formation. The polyurethane (PU)/DMSO spinning solution was injected into the core flow channel while the nonsolvent (deionized water: $\mathrm{DMSO}=5: 1(\mathrm{v}, \mathrm{v})$ ) was injected 
into the symmetrical sheath flow channels. The fiber gel can be solidified at the coaxial flow channel based on the principle of nonsolvent induced phase separation. In details, when the dispersed PU molecular chains meet with nonsolvent in the coaxial flow channel, the rapid diffusion between deionized water and DMSO solvent causes the aggregation of PU chains and leads to phase-separation from DMSO and the macroscopic fibers. ${ }^{19}$ Meanwhile, the "hydrodynamic focusing and alignment" effect results in the polymer molecules aligning along the flow direction. ${ }^{20,21}$ The as-prepared MPPU fibers can be continuously wound onto the roller (Figure S1c) and dried before the collection of fibers (Figure 1c). The as-obtained MPPU fibers can be facilely wove into lightweight fabrics $\left(90.6 \mathrm{~g} / \mathrm{m}^{2}\right.$, see Table $\mathbf{S 1}$ for details) with plain structure, approximately 30\% lighter than cotton fabric with the same thickness (Figure 1d) and the fibers are interlaced tightly from warp and weft (Figure 1e), indicating the fibers could be continuous and mass-produced with good spinnability. The MPPU fibers exhibit smooth surface appearance (Figure 1f) and internal multiscale disordered porous structure (Figure 1g). The radial cross-section view shows that several large cavities can separate PU polymer skeletons, and the higher magnification (Figure 1h, i) shows that small pores (nano- and micro-pores) are embedded in PU polymer skeletons. 


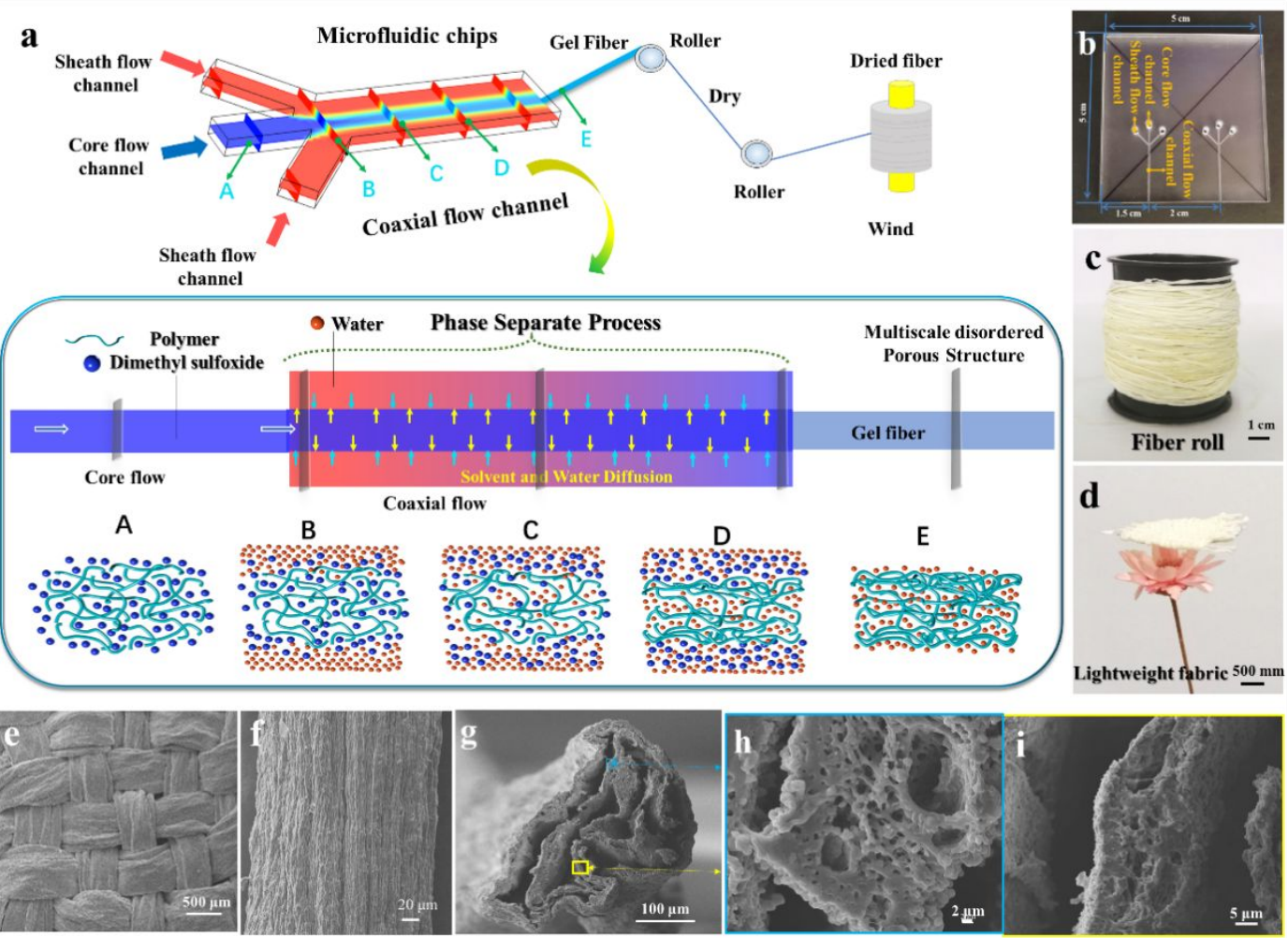

Figure 1. Schematic illustration of multiscale disordered porous elastic fibers fabrication. a) The microfluidic spinning process and the mechanism of fiber formation. b) The optical images of the microfluidic chips and the channel patterns. c) The collected MPPU fibers and d) the lightweight woven fabrics with plain structures. e) SEM images of the woven fabrics and f) MPPU fibers from the longitudinal view, g-i) the radial cross-section under different magnifications.

The forming mechanism of multiscale disordered porous structures with large cavities and small pores are illustrated in the radial direction (Figure 2a). In the phase separating process, once the nonsolvent access the spinning solution hybrid, the first stage is the rapid large-scale separation of PU/DMSO spinning solution, fast and vast water stream into spinning solution and thus lead to the formation of large cavities ultimately. Afterwards, the second stage is the slowly penetrating process, timeconsuming exchange of water and DMSO happens and induces the final formation of the multiscale disordered small pores. Therefore, after these two steps of phase separation, the nascent MPPU gel fibers are achieved followed by the dried porous fibers. 
In order to clarify the forming mechanism of the porous structures, we designed an experiment to characterize the tendency of fiber micro-structure. Along with the wet spinning process, fiber samples at five timescales $(0,30,150,600 \mathrm{~s}$ and dried) were collected and brittle broke by liquid nitrogen respectively, the cross-sectional SEM images is shown in Figure 2b. The fiber shape is preliminary formed as soon as the spinning solution injected into coagulation bath (timescale at $0 \mathrm{~s}$ ), but the phase separation does not ignite. For the gel fibers at 30s, the big cavities in the fiber core are gradually achieved, and the small pore at the fiber boundary are prioritized formed. For the gel fibers at $150 \mathrm{~s}$, the "cavity and small pores structure" is further configured, the large cavities shape up prior to the small pores based on an outside-to-inside rule along with the radial direction. After 600 s coagulation treatment, the gel fiber seems to possess full-blown porous structures, the small pores are multiscale in size and disordered in distribution. Compared with the gel fibers, the obtained dried fibers shrink into an irregular shape with smaller diameter and tight structure, in which cavities and micro-pores are also contracted. However, the multiscale disordered porous structure is still existent, the porosity of $\mathrm{MPPU}_{10}$ is about $63.6 \% \pm 0.6 \%$ and the pore size are multiple-peaked distribution spanning two order of magnitude, with broad distributions averaged at $\sim 469 \mathrm{~nm}$ for the nanopores and $\sim 34 \mu \mathrm{m}$ for the micropores, respectively (Figure 2c). The pore sizes are in the size range comparable with the wavelength of sunlight $(\sim 0.3-2.5 \mu \mathrm{m})$, which scatter sunlight strongly. The nanopores are also much smaller than the IR wavelength $(\sim 7-14 \mu \mathrm{m})$, so the MPPU fiber is still highly transparent to IR. Thus, MPPU fiber has advantages in IR transparency and body cooling property 
over common textile materials which strongly absorb human body radiation and have very low IR transparency (Figure 2d). This is further demonstrated by the high IR transmittance of the MPPU ${ }_{10}$ fabric measured with FTIR, which shows 97.1\% IR transmittance for wavelength 7 14 $\mu \mathrm{m}$, much greater than that of cotton and Lycra fabric (78.4\% for cotton, and $59.7 \%$ for Lycra, Figure $2 \mathbf{e}$ ), and the mechanism is consistent with some reported work. ${ }^{22-24}$ Typical stress-strain curves for MPPU fibers are shown in Figure 2f, MPPU fibers exhibit the gradient average tensile strength value from $70.4 \pm 0.5$ to $57.8 \pm 0.7 \mathrm{MPa}$, and the average elongation from $528 \pm 5 \%$ to $455 \pm$

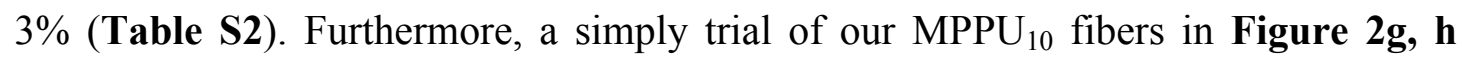
indicates the outstanding elongation performance with 500\% elongation. These results demonstrate that the as-prepared MPPU fibers possess good mechanical and elastic properties and facilitate to weave into functional textiles. 


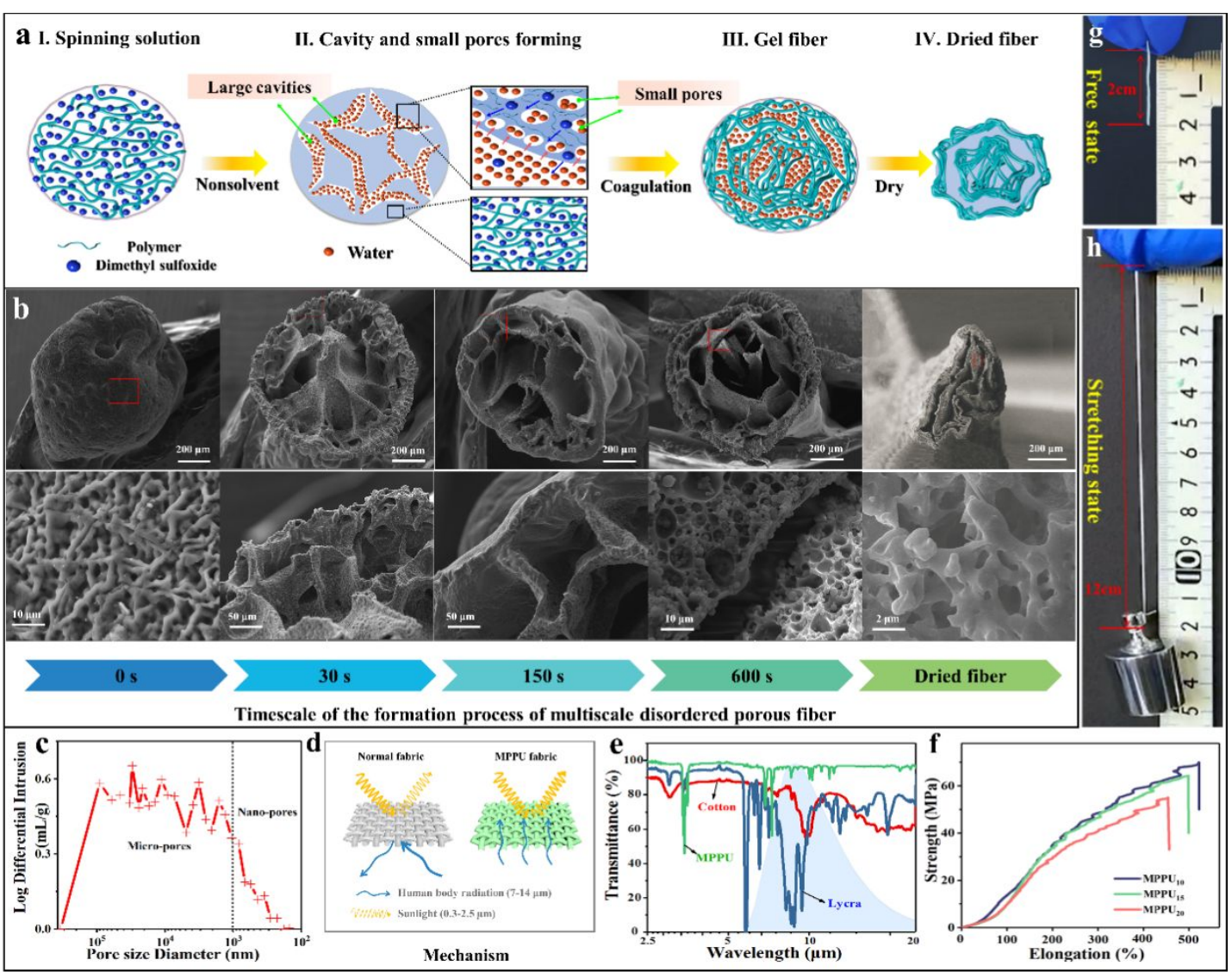

Figure 2. Mechanism of pores forming process and self-cooling effect of MPPU fibers. a) Schematic illustration of the forming process of porous structure in the radial direction. b) Timescale of the formation process of MPPU fiber and the cross-sectional SEM images in different time nodes. c) Size distributions of nano- and micro-pores of MPPU ${ }_{10}$ fibers. d) Schematics of comparison between normal fabric and MPPU fabric, where the MPPU is transparent to human body radiation. e) FTIR transmittance of cotton, Lycra and MPPU 10 fabrics. f) The mechanical properties of as-obtained elastic fibers. $g, h$ ) Stretching behavior for MPPU ${ }_{10}$ fibers under the weight loading of a $50 \mathrm{~g}$.

We further evaluated the self-cooling performance of the as-prepared fibers with multiscale disordered porous structure. Fibers $\left(\mathrm{MPPU}_{10}, \mathrm{MPPU}_{15}, \mathrm{MPPU}_{20}\right.$, cotton, commercial Lycra) and their woven fabrics were compared by placing them under the same heating stage. The corresponding infrared thermal (IR) images were recorded under the stage temperature $30,45,60{ }^{\circ} \mathrm{C}$, respectively (Figure 3a, b), the results indicated that $\mathrm{MPPU}_{10}$ exhibits the most similar temperature with the stage temperature by the lowest color discrimination, which implying the best heat dissipation property among the samples. Furthermore, the real-time temperature curves of these fabrics 
during the heating $\left(20 \sim 40{ }^{\circ} \mathrm{C}\right)$ and cooling $\left(40 \sim 20{ }^{\circ} \mathrm{C}\right)$ was illustrated in Figure 3c and 3d respectively. The MPPU ${ }_{10}$ exhibits much rapidly heating and cooling capacities in stage temperature rising/falling process (Figure 3c, d). The temperature difference $(|\Delta T|)$ between the twisted fibers or woven fabrics and the stage also illustrated in Figure 3e and 3f respectively. The results indicated that $|\Delta T|$ of $\operatorname{MPPU}_{10}$ fabrics is at the lower level $2.5{ }^{\circ} \mathrm{C}$ compared with $|\Delta T|$ of cotton and Lycra at $5.3{ }^{\circ} \mathrm{C}$ and $5.7{ }^{\circ} \mathrm{C}$ in $45{ }^{\circ} \mathrm{C}$ stage temperature, respectively (Figure 3f). Skin temperature experiments in Figure 3g further demonstrates that MPPU fabrics have a better cooling effect than traditional fabrics. For the single layer fabrics, the temperature difference between MPPU and other samples are at least $2.5^{\circ} \mathrm{C}$ (Figure 3g2); MPPU fabrics with different layer thickness $(1,2,4$ layers) exhibits the lowest color discrimination from the corresponding IR images (Figure 3g3), and the smallest temperature difference (Figure 3g4). Although the temperature difference gradually increases with the increasing thickness of fabric, implying the multi-layer structure could hinder the heat dissipation and weaken cooling effect, MPPU has an obvious cooling effect than normal fabrics. The "QU" shaped metallic pattern is applied to visual present IR transmittance of fabrics through thermal imaging (Figure 3h), and only bare skin and $\mathrm{MPPU}_{10}$ specimens can clearly show the 'QU' pattern. Meanwhile, the surface temperature of cotton and Lycra appear colder than that of $\mathrm{MPPU}_{10}$ due to their poorer heat dispersion, the average surface temperature of $\mathrm{MPPU}_{10}$, cotton and Lycra are $33.9^{\circ} \mathrm{C}, 32.3^{\circ} \mathrm{C}$ and $31.6^{\circ} \mathrm{C}$, respectively, according to the IR image..$^{25}$ Such a temperature difference indicates $\mathrm{MPPU}_{10}$ fabrics possess excellent heat dissipation property. 

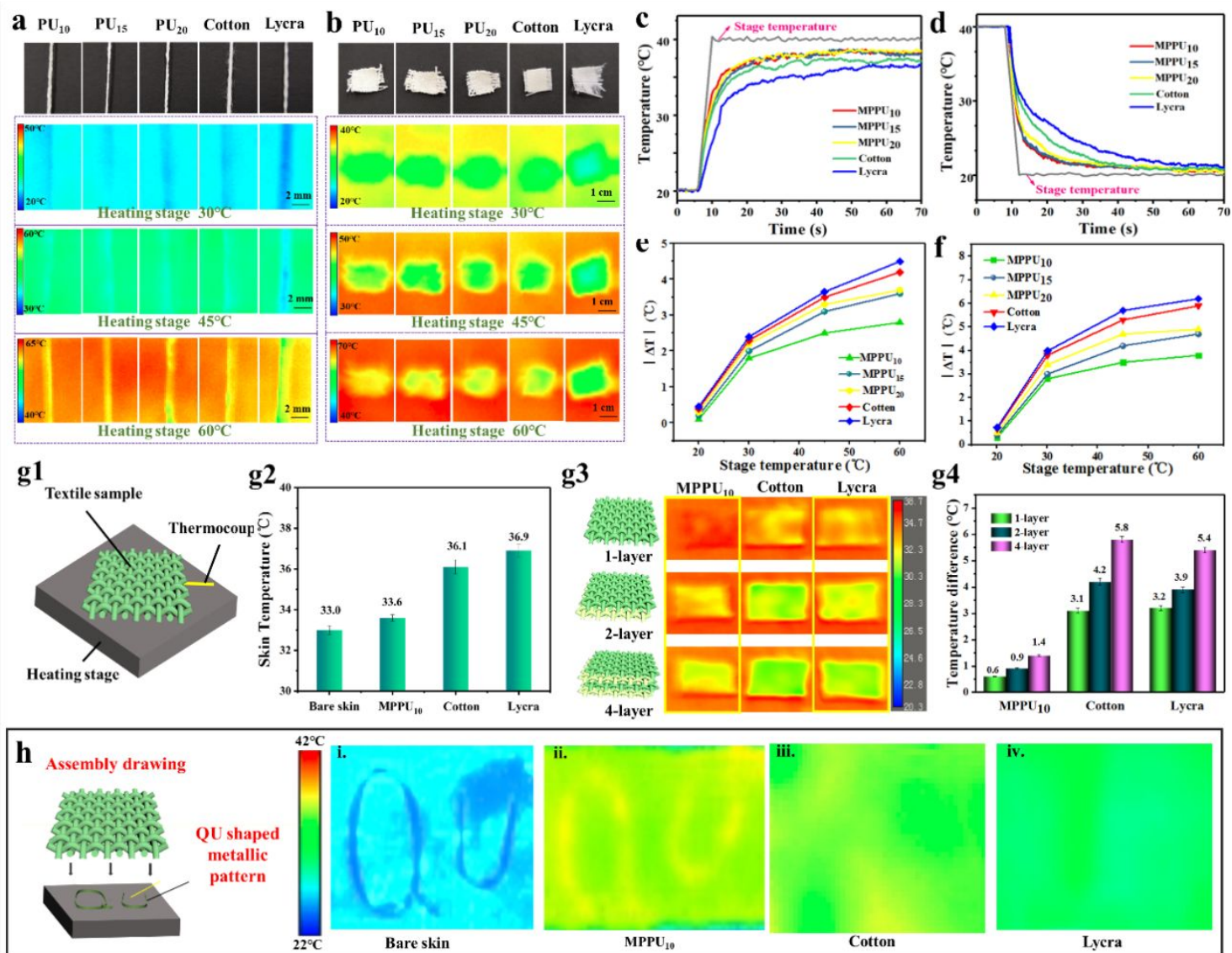

Figure 3. Self-cooling performance of MPPU fibers. a, b) Self-cooling behaviors of MPPU ${ }_{10}, M_{P P U_{15}}$, $\mathrm{MPPU}_{20}$, cotton, commercial Lycra, respectively under temperatures of 30,45 and $60{ }^{\circ} \mathrm{C}$, where environment temperature is $28^{\circ} \mathrm{C}$. c, d) Temperature curves of different fabrics under dynamic heating/cooling between $20^{\circ} \mathrm{C}$ and $40^{\circ} \mathrm{C}$. e, f) The absolute temperature difference $(|\Delta \mathrm{T}|)$ between the twisted fibers or woven fabrics and the background stage. g1,2) Schematic illustration of the experimental setup of textile thermal measurement and corresponding thermal measurement of bare skin, MPPU $_{10}$, cotton and Lycra. g3) Self-cooling behaviors of MPPU 10 , cotton, and commercial Lycra for 1, 2, 4 layers of woven fabrics under temperatures of $37{ }^{\circ} \mathrm{C}$ and $\mathrm{g} 4$ ) the corresponding temperature differences between stage temperature and samples' surface temperature. h) Thermal IR images of bare skin and the MPPU 10 , cotton, commercial Lycra.

In addition to self-cooling function, autonomous sensations are also essential for

flexible wearable sensors applied on smart clothing. As a proof of concept, graphene conductive inks are decorated onto MPPU fibers to gain sensing property via capillaryassisted dip-coating process (Figure 4a). The SEM images of the as-achieved graphene modified MPPU (G@MPPU) fibers show that graphene nanosheets uniformly deposit on the surface of MPPU fiber, and from the cross section view of G@MPPU fiber, the cavities and micropores do not affect by the deposition of graphene implying the 
available intrinsic self-cooling performance of G@MPPU fiber. Besides, G@MPPU fiber can be facilely stretched to $200 \%$, even $400 \%$, with outstanding stretchable capacity (Figure 4b).

The strain sensing performance of G@MPPU fiber were investigated. The relative resistance variation $\left(\Delta R / R_{0}\right)$ in Figure 4c demonstrated that G@MPPU ${ }_{10}$ fibers exhibit highly sensitive behavior with $\Delta R / R_{0}=15$ under $10 \%$ strain. ${ }^{26,27}$ Gauge factor (GF, GF $\left.=\left(\Delta R / R_{0}\right) / \Delta \varepsilon\right)$ in Figure 4d present that GF of G@MPPU $\mathrm{M}_{10}$ fiber increased markedly under the large tensile strain (more than 50\%) and increased gently under the small tensile strain (less than 50\%). The average high GF at $0.5 \%$ tensile strain endows a desirable high sensitivity at small strain and broad sensing range of such sensor for greatly potentials in full-range human motion recording.

The detection limit of G@MPPU ${ }_{10}$ fiber was demonstrated by applying gradually decreasing step strain (Figure 4e). Benefiting from the good sensitivity at small strain range, the detection limit could be as minute as $0.5 \%$, and the output signal was highly reproducible at minute $0.5 \%$ strain with $\Delta R / R_{0}=1.3$ (inset in Figure 4e). The fast response undoubtedly contributes to the real-time monitoring of fast and intricate movements for users, such as yoga and dance. Durability and stability of the G@MPPU fiber are investigated through periodic stretching/releasing cycles from $0 \%$ to $10 \%$ strain over 3000s with $2 \mathrm{~Hz}$ frequency (Figure 4g). After 6000 times cycling, the relative signal drift at 10\% strain of G@MPPU fiber sensor was -8.3\% (based on the first cycle), allowing for the reliable practical utilization as daily wearable sensors. The strain sensing mechanism could be explained by the connection change of the 
overlapped graphene nanosheets on the fiber surface as schematic diagram in Figure 4h. ${ }^{28}$ Stretching and releasing of the PU polymer matrix in the fiber results in disconnection and recovery of the overlapped area of the graphene nanosheets, which changes the resistance of G@MPPU fiber.
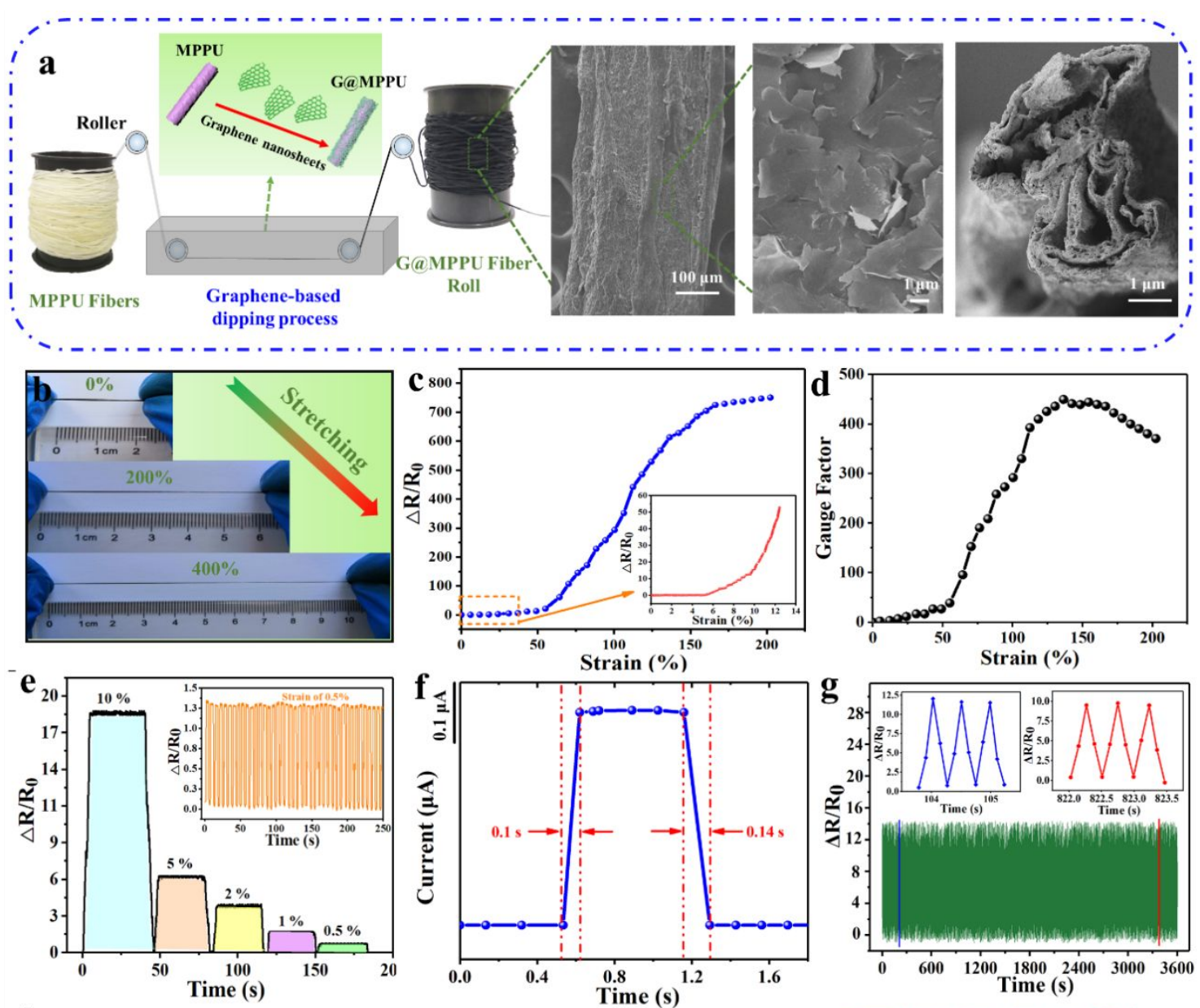

h

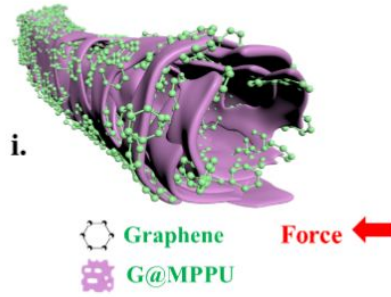

ii.

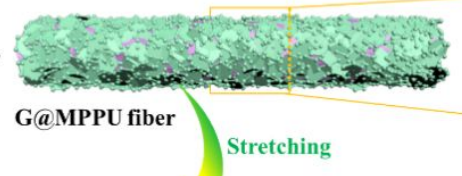

iii.

Stretched fiber

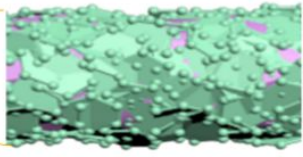

Figure 4. Strain performance of G@MPPU fibers. a) Schematic of the graphene capillary-assisted dip-coating process for fabricating G@MPPU fibers. b) Tensile capacity of G@MPPU 10 fibers under loads of $200 \%$, and $400 \%$. c) Relative resistance variation of G@MPPU 10 fibers along with increasing strain. The inset images show the relative resistance variation of the fibers at the tensile strains within the range of $0 \%-20 \%$. d) Gauge factor variation of the G@MPPU $U_{10}$ fibers under $200 \%$ strain. e) Relative resistance variation under gradually decreasing step strain from $10 \%$ to $0.5 \%$ strain. The inset image 
shows the durability of fibers under $0.5 \%$ strain. f) Response time of the G@MPPU 10 fiber stretched and released at a step strain of 5\%. g) Stability of a G@MPPU $U_{10}$ fiber $(5 \mathrm{~cm})$ from $0 \%$ strain to 10\% strain over 3000s under a frequency of $2 \mathrm{~Hz}$. The inset shows the response signal at 3 consecutive input. h) Schematic diagram of the surface graphene nanosheets distribution before and after stretching force of G@MPPU ${ }_{10}$ fiber, which indicate its simplified conductive mechanism.

The temperature sensing performance of the $\mathrm{G} @ \mathrm{MPPU}_{10}$ fabrics was also monitored under a heating stage as shown in Figure 5. The G@MPPU ${ }_{10}$ sensor exhibits a TCR of $-0.815 \% /{ }^{\circ} \mathrm{C}$ in range of $20 \sim 100^{\circ} \mathrm{C}$, which indicates its superb temperature sensitivity (Figure 5a). As expected, graphene modified MPPU fibers exhibited negative TCR behavior due to the variable-range hopping mechanism, which facilitates the charge carrier mobility with increasing temperature. ${ }^{29}$ We also carried out a facile experiment to monitor the temperature changes from human skin surface by attaching G@MPPU 10 sensor on volunteer's forearm. The skin temperature increases up to $41.2^{\circ} \mathrm{C}$ after $10 \mathrm{~s}$ with the thermos from the normal skin temperature $31.5^{\circ} \mathrm{C}$ (Figure $\mathbf{5 b}$ and $\mathbf{c}$ ). Relative resistance changes with time by heating and cooling from 25 to $45^{\circ} \mathrm{C}$ implies that two symmetrical curves of resistance and temperature shows highly consistent with each other (Figure 5d). From the magnified frame in Figure 5d, the hysteresis of resistance changes at the turning point of temperature exhibit negligible lag time $\sim 0.4 \mathrm{~s}$, which implies the margin effect of thermal hysteresis on the fabric sensor (Figure 5e). 

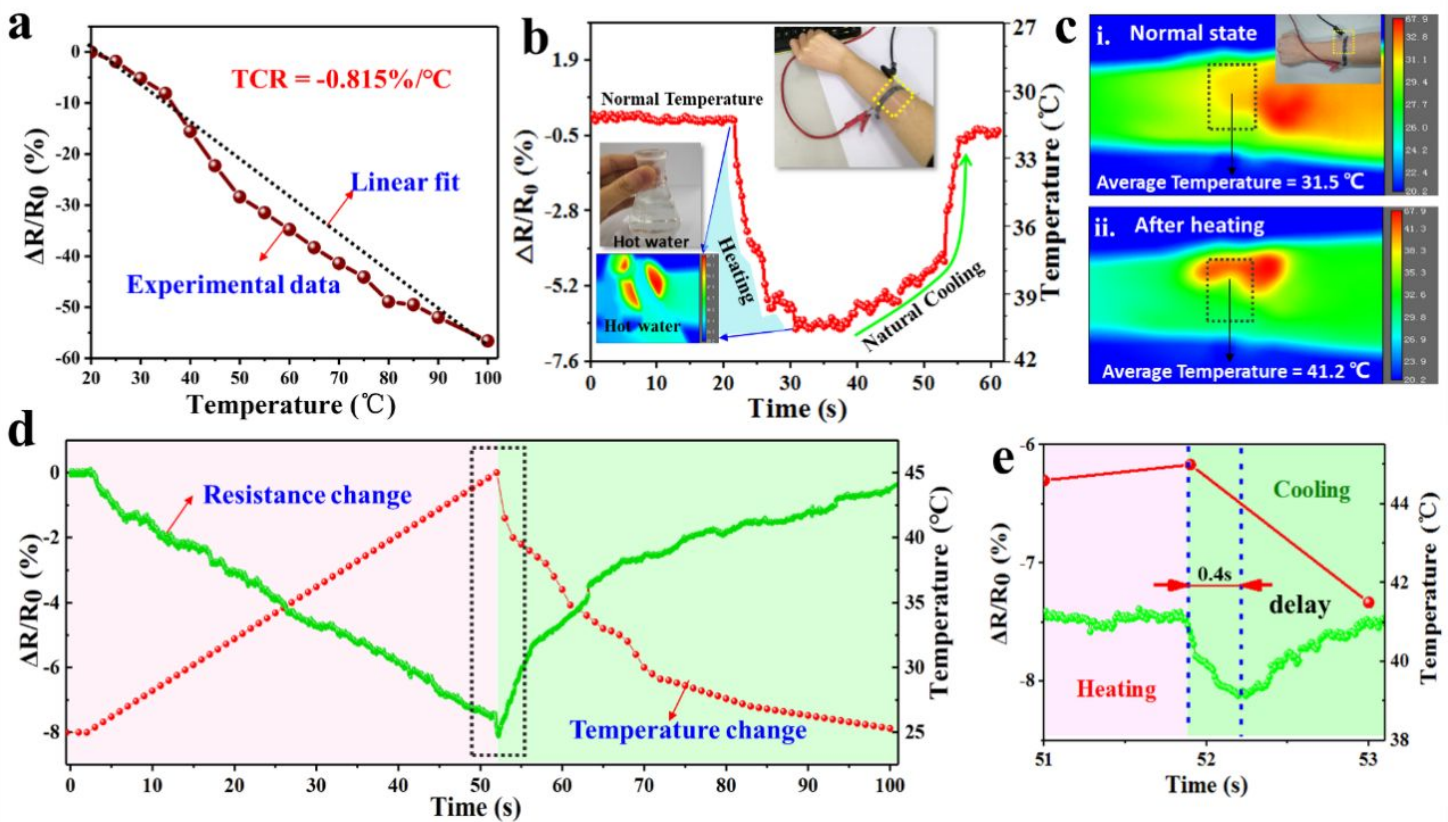

Figure 5. Temperature performance of G@MPPU fibers. a) Relative resistance changes of $\mathrm{G} @ \mathrm{MPPU}_{10}$ fibers upon increasing temperature from $20^{\circ} \mathrm{C}$ to $100^{\circ} \mathrm{C}$. b) Forearm skin temperature at normal state and after putting of a thermos $\left(60^{\circ} \mathrm{C}\right)$, insets are the optical image of testing, and the IR image when putting the thermos on the forearm. c) IR images of the forearm before and after putting of the thermos. d) Relative resistance changes as a function of time with temperature heating and cooling from 25 to $45^{\circ} \mathrm{C}$. This temperature range can cover the human body temperatures, indicating the temperature sensing capability of the G@MPPU textiles. e) The hysteresis of resistance changes at the turning point of temperature (the marked frame in Figure 5d).

We also employed MPPU and G@MPPU fibers to fabricate an integrated smart sportswear. As demonstrated in Figure 6a, nine possible sensation zones are designed on the exemplary smart clothing, which are noted as sensor A (neck monitoring), B (shoulder monitoring), $\mathrm{C}$ (chest monitoring), D (waist monitoring), E (wrist monitoring), and $\mathrm{F}$ (finger monitoring), respectively. Subtle physiological signal capturing and limb movements could be recorded and discriminated via sensor A-F alone or in combination as shown in Figure 6b-j. At the neck position, human swallowing and speaking activity can be monitored by sensor A (Figure $\mathbf{6 b}, \mathbf{c}$ ), exhibiting characterized patterns with good stabilization. Sensor B located at human shoulder can detect the stretching exercise and walking motion (Figure 6d, e, Figure 
S2) with different signal display. Furthermore, the chest respiration and chest expanding recorded by sensor $\mathrm{C}$, allowing for the real-time monitoring of respiration rate and depth in relaxation and after exercise (Figure 6f, g). Waist-related motions such as side twist can be recorded by Sensor D (Figure 6h), and pulse current signal recorded by sensor E (60-65 beats/minutes) under normal conditions, as well as the finger bending activity recorded by sensor F (Figure 6i, j). Once wearing such smart sportswear, a full-range detection of human activities can be facilely monitored and recorded.

For practical application, a wireless system is established as in Figure 6k, the external connections of the sensors include Bluetooth module, battery/portable power source, and mobile phone (details see Figure S3). An APP in the phone can real-time monitor the resistance variation. For the proof of concept, a demo of smart glove is designed (Figure 61, VIDEO R1), the index finger bending test is carried out and the resistance variation curves in monitoring of finger bending movements show good sensitivity and effectivity of G@MPPU sensors (Figure 6m). 

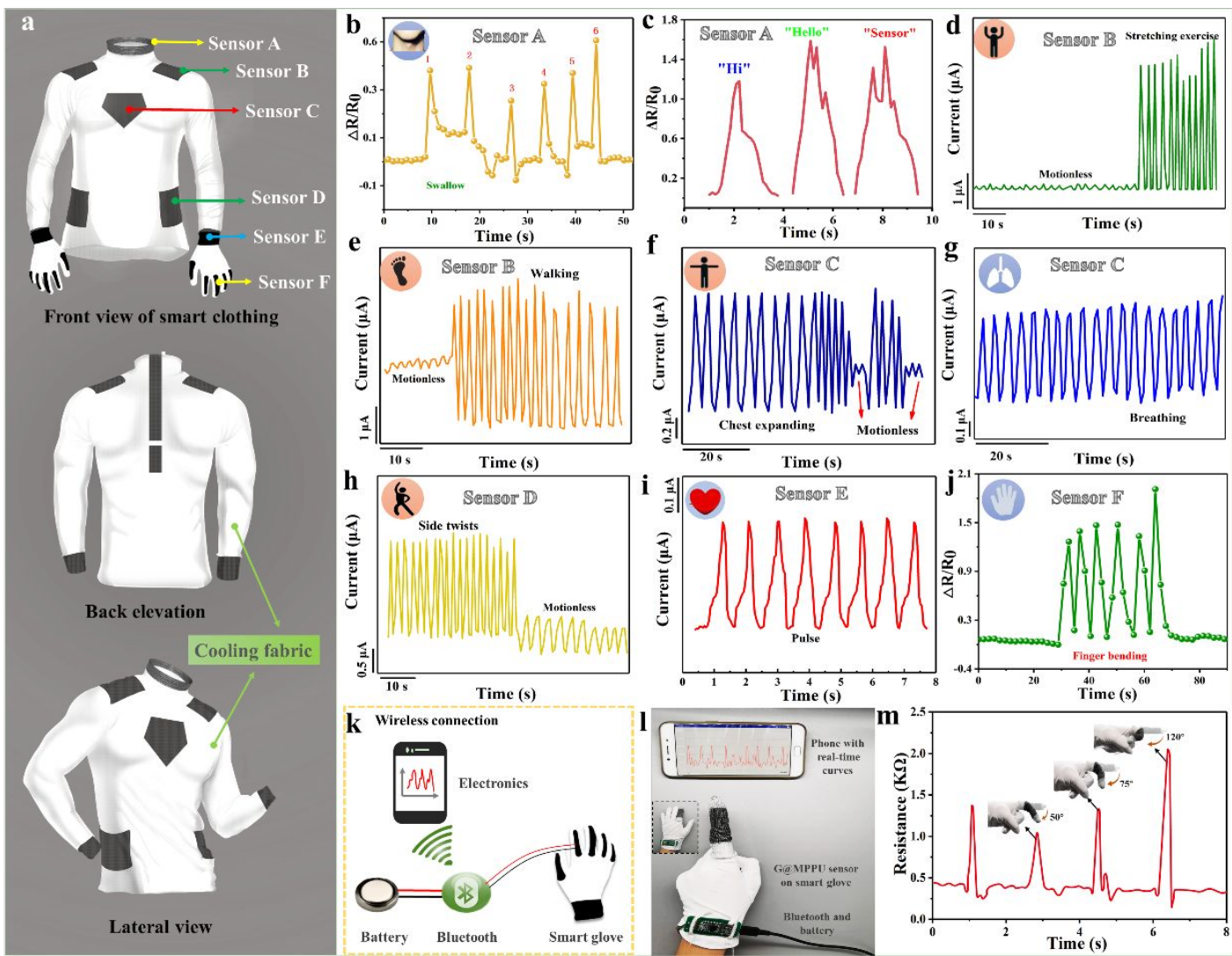

Figure 6. Smart clothing and applications on movements and vital signals monitoring. a) The front, back and lateral view of the exemplary integrated smart clothing, which is fabricated with MPPU fibers for body cooling (white part) and G@MPPU fibers for sensations (black part). There are nine sensation zones designed on the exemplary clothing. Responsive curves of G@MPPU sensor in monitoring of b) swallow, c) speaking, d) stretching exercise, e) walking, f) chest expanding, g) breathe, h) side twist, i) pulse, and j) finger bending. $\mathrm{k}, 1$ ) Schematic and optical images of the external connections of the fabric sensors with wireless connection $\mathrm{m}$ ) and the data received through APP on the phone for various finger bending movements.

\section{CONCLUSIONS}

In summary, we present a multiscale disordered porous polyurethane fiber with good self-cooling property that can be applied in personal thermal management. After the capillary-assisted adsorption of graphene inks, the modified porous fibers could also possess real-time strain and temperature sensing with high GF and TCR. As a proof of concept, the integrated smart sportswear is constructed with MPPU fibers for body cooling and G@MPPU fibers for sensations that can actively measure body temperature and monitor the large-scale limp movement and subtle human physiological signals as 
well as the intrinsic self-cooling capacity.

\section{EXPERIMENTAL SECTION}

Fabrication of MPPU fibers. Graphene $(G)$ was prepared from the purified natural graphite by the modified Hummer's method described previously. ${ }^{30-33}$ For preparing MPPU fibers, a poly (methyl methacrylate) (PMMA) plate with slit channel was fabricated using standard micro carving methods (Figure S1a) and sealed with another intact PMMA plate. The three inlet channels have a square cross-section (side length: $h=300 \mu \mathrm{m}$ ), whereas the cross-section of coaxial channel is rectangle (side length: $h=300 \mu \mathrm{m}, w=600 \mu \mathrm{m})$, as seen in Figure S1b. Two solutions were introduced from the inlets: the spinning solution of polyurethane (PU, polymer) dissolved in dimethyl sulfoxide (DMSO, solvent) was chosen as core fluid with PU concentration of 10, 15 and 20 wt.\%; and deionized water was chosen as the outer sheath channel for phase separation, as well as the coagulation bath. The flow rate of inlets was set to the same value for all the fiber fabrication process. Typically, the sheath flow rate was fixed at $10 \mathrm{~mm}^{3} \mathrm{~s}^{-1}$ and the core flow rate was $20 \mathrm{~mm}^{3} \mathrm{~s}^{-1}$. All the fibers were prepared at the same flow speed with the same microfluidic chip, and the as-prepared fibers are noted as $\mathrm{MPPU}_{10}, \mathrm{MPPU}_{15}, \mathrm{MPPU}_{20}$ corresponding to spinning solution with different ratios. After coagulation, the fibers were washed by deionized water and then rolled onto the collecting tube as illustrated in Figure $\mathbf{2 b}$, and the finally dried winding MPPU fiber roll were shown in Figure 2c and the fabrication process is shown in Video S2.

Fabrication of G@MPPU fibers. Homemade graphene solution was firstly treated by ultra-sonication $(600 \mathrm{~W})$ for $15 \mathrm{~min}$ and then dispersed in the waterborne polyurethane solution (1 wt.\%) with the concentration about $25 \mathrm{mg} / \mathrm{mL}$. As illustrated in Figure 5a, the MPPU fiber was unwound from the fiber roll and guide by the roller to the dipcoating device for $10 \mathrm{~min}$ at room temperature. Then the graphene treated MPPU fibers was dried at room temperature and wound to a new fiber roll.

Characterization on structural features. The morphologies and microstructures of MPPU and G@MPPU fibers were investigated through scanning electron microscope (SEM, JEOL JSM-840, Japan). Structural parameters, such as pore size and porosity, 
were measure on an Auto Pore IV 9500 Automatic Mercury Porosimeter (Micromeritics Instrument Corp, USA).

Characterization on Infrared reflectance. Spectra were measured using a Nicolet 5700 FT-IR spectrometer (Thermo Nicolet Corp, USA), the wave number range was $4000-500 \mathrm{~cm}^{-1}$ with a resolution of $4 \mathrm{~cm}^{-1}$. All spectra are the average of 32 scans at the room temperature.

Characterization on mechanical properties. The tensile test was performed using a single fiber electronic tensile strength tester in accordance with ISO5079 standard. Each specimen was tested ten times, and the average values were reported. The cross section of each fiber was assumed to be circular and was determined with an optical microscope. Characterization on thermal properties. An IR thermal camera (FLIR, A300 series, USA) was used to capture the temperature profile of specimens under different stage temperature conditions. The working distance was $\sim 25 \mathrm{~cm}$. The specimen's temperature was monitored and recorded by a thermal couple connected to a temperature controller (Taiwan TES Co., Ltd, China). The ambient temperature is kept at $\sim 24^{\circ} \mathrm{C}$. The IR images were taken when the surface temperature was stable. The thermal conductivity of the MPPU textile was estimated by a thermal conductivity meter (Shanghai KAnalysis Co., Ltd, China).

Characterization on sensing properties. The resistance of specimens was obtained by a parameter analyzer (4200A-SCS, Keithley, USA), and the parameters were set at the voltage bias model, voltage level of $0.1 \mathrm{~V}$, auto voltage range, measurements of 1000 , and delay of $0.1 \mathrm{~s}$.

\section{ASSOCIATED CONTENT}

\section{Supporting Information.}

Supporting Figures S1-S2, the descriptions of the microfluidic chip and as-prepared MPPU fibers, the measurement of the G@MPPU fabric sensor on the knee. Supporting Tables S1-S2, grams per square meter of MPPU and traditional fabrics, pore size and distributions of MPPU fibers with different PU weight ratios. Supporting Video S2, the 
fabrication process of the MPPU fibers.

\section{ACKNOWLEDGEMENTS}

Financial support of this work was provided by Natural Science Foundation of China via grant No. 51672141 and 51306095, Natural Science Foundation of Shandong Province of China (ZR2018QEM004), Research and Development Program of Shandong Province of China (grant number 2019GGXI02022, 2019JZZY010340 and 2019JZZY010335).

\section{REFERENCES}

1. Kim, J.; Campbell, A. S.; de Ávila, B. E.-F.; Wang, J. Wearable Biosensors for Healthcare Monitoring. Nat. Biotechnol. 2019, 37,389-406.

2. Gao, W.; Emaminejad, S.; Nyein, H. Y. Y.; Challa, S.; Chen, K.; Peck, A.; Fahad, H. M.; Ota, H.; Shiraki, H.; Kiriya, D. Fully Integrated Wearable Sensor Arrays for Multiplexed In Situ Perspiration Analysis. Nature 2016, 529, 509514.

3. Wu, X.; Peng, H. Polymer-Based Flexible Bioelectronics. Sci. Bull. 2019, 64, 634-640.

4. Weng, W.; Chen, P.; He, S.; Sun, X.; Peng, H. Smart Electronic Textiles. Angew. Chemie Int. Ed. 2016, 55, 6140-6169.

5. Yamada, T.; Hayamizu, Y.; Yamamoto, Y.; Yomogida, Y.; Izadi-Najafabadi, A.; Futaba, D. N.; Hata, K. A Stretchable Carbon Nanotube Strain Sensor for Human-Motion Detection. Nat. Nanotechnol. 2011, 6, 296-301.

6. Sehrawat, P.; Abid; Islam, S. S.; Mishra, P. Reduced Graphene Oxide Based Temperature Sensor: Extraordinary Performance Governed by Lattice Dynamics Assisted Carrier Transport. Sensors Actuators, B Chem. 2018, 258, 424-435.

7. Trung, T. Q.; Duy, L. T.; Ramasundaram, S.; Lee, N. E. Transparent, Stretchable, and Rapid-Response Humidity Sensor for Body-Attachable Wearable Electronics. Nano Res. 2017, 10, 2021-2033.

8. Amjadi, M.; Kyung, K. U.; Park, I.; Sitti, M. Stretchable, Skin-Mountable, and Wearable Strain Sensors and Their Potential Applications: A Review. Adv. Funct. Mater. 2016, 26, 1678-1698.

9. Mattmann, C.; Clemens, F.; Tröster, G. Sensor for Measuring Strain in Textile. Sensors 2008, 8, 3719-3732.

10. Stoppa, M.; Chiolerio, A. Wearable Electronics and Smart Textiles: A Critical Review. Sensors (Switzerland) 2014, 14, 11957-11992.

11. Post, E. R.; Orth, M.; Russo, P. R.; Gershenfeld, N. E-Broidery: Design and Fabrication of Textile-Based Computing. IBM Syst. J. 2000, 39, 840-820. 
12. Cheng, Y.; Wang, R.; Sun, J.; Gao, L. A Stretchable and Highly Sensitive Graphene-Based Fiber for Sensing Tensile Strain, Bending, and Torsion. Adv. Mater. 2015, 27, 7365-7371.

13. Eltzner, B.; Wollnik, C.; Gottschlich, C.; Huckemann, S.; Rehfeldt, F. The Filament Sensor for near Real-Time Detection of Cytoskeletal Fiber Structures. PLoS One 2015, 10, e0126346.

14. Zhao, H.; Zhang, Y.; Bradford, P. D.; Zhou, Q.; Jia, Q.; Yuan, F.-G.; Zhu, Y. Carbon Nanotube Yarn Strain Sensors. Nanotechnology 2010, 21, 305502.

15. Choudhary, T.; Rajamanickam, G. P.; Dendukuri, D. Woven Electrochemical Fabric-Based Test Sensors (WEFTS): A New Class of Multiplexed Electrochemical Sensors. Lab Chip 2015, 15, 2064-2072.

16. Paradiso, R.; Loriga, G.; Taccini, N. A Wearable Health Care System Based on Knitted Integrated Sensors. IEEE Trans. Inf. Technol. Biomed. 2005, 9, 337344.

17. Luo, S.; Liu, T. SWCNT/Graphite Nanoplatelet Hybrid Thin Films for SelfTemperature- Compensated, Highly Sensitive, and Extensible Piezoresistive Sensors. Adv. Mater. 2013, 25, 5650-5657.

18. Li, J.; Xu, B. Novel Highly Sensitive and Wearable Pressure Sensors from Conductive Three-Dimensional Fabric Structures. Smart Mater. Struct. 2015, 24,125022 .

19. Hołda, A. K.; Vankelecom, I. F. J. Understanding and Guiding the Phase Inversion Process for Synthesis of Solvent Resistant Nanofiltration Membranes. J. Appl. Polym. Sci. 2015, 132, 1-17.

20. Xin, G.; Zhu, W.; Deng, Y.; Cheng, J.; Zhang, L. T.; Chung, A. J.; De, S.; Lian, J. Microfluidics-Enabled Orientation and Microstructure Control of Macroscopic Graphene Fibres. Nat. Nanotechnol. 2019, 14, 168.

21. Hu, X.; Tian, M.; Sun, B.; Qu, L.; Zhu, S.; Zhang, X. Hydrodynamic Alignment and Microfluidic Spinning of Strength-Reinforced Calcium Alginate Microfibers. Mater. Lett. 2018, 230, 148-151.

22. Chen, T.; Qiu, L.; Yang, Z.; Cai, Z.; Ren, J.; Li, H.; Lin, H.; Sun, X.; Peng, H. An Integrated "Energy Wire" for Both Photoelectric Conversion and Energy Storage. Angew. Chemie Int. Ed. 2012, 51, 11977-11980.

23. Wang, L.; Wang, L.; Zhang, Y.; Pan, J.; Li, S.; Sun, X.; Zhang, B.; Peng, H. Weaving Sensing Fibers into Electrochemical Fabric for Real-Time Health Monitoring. Adv. Funct. Mater. 2018, 28, 1804456.

24. Cai, L.; Song, A. Y.; Li, W.; Hsu, P. C.; Lin, D.; Catrysse, P. B.; Liu, Y.; Peng, Y.; Chen, J.; Wang, H.; Xu, J.; Yang, A.; Fan, S.; Cui, Y. Spectrally Selective Nanocomposite Textile for Outdoor Personal Cooling. Adv. Mater. 2018, 30, $1-7$.

25. Hsu, P.-C.; Song, A. Y.; Catrysse, P. B.; Liu, C.; Peng, Y.; Xie, J.; Fan, S.; Cui, Y. Radiative Human Body Cooling by Nanoporous Polyethylene Textile.

Science (80-. ). 2016, 353, 1019-1023.

26. White, M. S.; Kaltenbrunner, M.; Głowacki, E. D.; Gutnichenko, K.; Kettlgruber, G.; Graz, I.; Aazou, S.; Ulbricht, C.; Egbe, D. A. M.; Miron, M. C. 
Ultrathin, Highly Flexible and Stretchable PLEDs. Nat. Photonics 2013, 7, 811.

27. Amjadi, M.; Pichitpajongkit, A.; Lee, S.; Ryu, S.; Park, I. Highly Stretchable and Sensitive Strain Sensor Based on Silver Nanowire-Elastomer Nanocomposite. ACS Nano 2014, 8, 5154-5163.

28. Chen, L.; Weng, M.; Zhou, P.; Huang, F.; Liu, C.; Fan, S.; Zhang, W. Graphene-Based Actuator with Integrated-Sensing Function. Adv. Funct. Mater. 2018, 1806057, 1-9.

29. Amjadi, M.; Sitti, M. Self-Sensing Paper Actuators Based on Graphite-Carbon Nanotube Hybrid Films. Adv. Sci. 2018, 5, 1-8.

30. Hu, X.; Tian, M.; Pan, N.; Sun, B.; Li, Z.; Ma, Y.; Zhang, X.; Zhu, S.; Chen, Z.; Qu, L. Structure-Tunable Graphene Oxide Fibers via Microfluidic Spinning Route for Multifunctional Textile. Carbon N. Y. 2019.

31. Hao, Y.; Tian, M.; Zhao, H.; Qu, L.; Zhu, S.; Zhang, X.; Chen, S.; Wang, K.; Ran, J. High Efficiency Electrothermal Graphene/Tourmaline Composite Fabric Joule Heater with Durable Abrasion Resistance via a Spray Coating Route. Ind. Eng. Chem. Res. 2018, 57, 13437-13448.

32. Lu, Y.; Tian, M.; Sun, X.; Pan, N.; Chen, F.; Zhu, S.; Zhang, X.; Chen, S. Highly Sensitive Wearable 3D Piezoresistive Pressure Sensors Based on Graphene Coated Isotropic Non-Woven Substrate. Compos. Part A Appl. Sci. Manuf. 2019, 117, 202-210.

33. Tian, M.; Lu, Y.; Qu, L.; Zhu, S.; Zhang, X.; Chen, S. A Pillow-Shaped 3D Hierarchical Piezoresistive Pressure Sensor Based on Conductive Silver Components-Coated Fabric and Random Fibers Assembly. Ind. Eng. Chem. Res. 2019, 58, 5737-5742. 


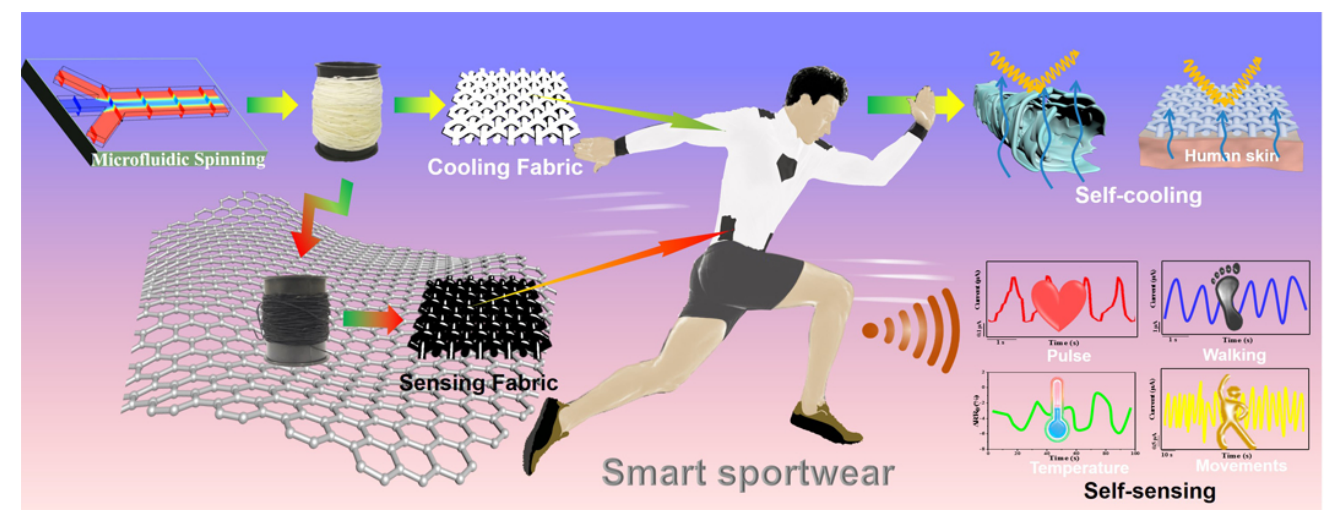

Smart sportswear can achieve self-sensing and self-cooling performances, which was constructed by fibers fabricated via the continuous microfluidic spinning and the post processing of graphene. Such smart clothes show great potential in human body monitoring and self-adaption.

$90 \times 34 \mathrm{~mm}(300 \times 300 \mathrm{DPI})$ 